\title{
Effect of different electrolytes on the swelling properties of calyx[4]pyrrole-containing polyacrylamide membranes
}

\author{
Artur J.M. Valente ${ }^{\mathrm{a}, *}$, Abílio J.F.N. Sobral ${ }^{\mathrm{a}}$, Alfonso Jiménez ${ }^{\mathrm{b}}$, Sílvia Patachia ${ }^{\mathrm{c}}$, \\ Ana R.C.B. Oliveira ${ }^{\text {a }}$, Victor M.M. Lobo ${ }^{\text {a }}$ \\ ${ }^{a}$ Department of Chemistry, University of Coimbra, 3004-535 Coimbra, Portugal \\ ${ }^{\mathrm{b}}$ Department of Analytical Chemistry, Nutrition and Bromatology, University of Alicante, P.O. Box 99, 03080 Alicante, Spain \\ ' Department of Chemistry, "Transilvania" University of Brasov, 29 Eroilor Str., 500036 Brasov, Romania
}

Received 5 March 2006; received in revised form 26 March 2006; accepted 2 April 2006

Available online 19 May 2006

\begin{abstract}
Calix[4]pyrrole (1) was synthesized and characterized and this macrocycle was incorporated in polyacrylamide gels. The presence of meso-octamethyl-porphyrinogen inside of gel was checked using infrared spectroscopy, differential scanning calorimetry, and swelling studies. The swelling degree of these hydrogels in equilibrium with different electrolytes $(\mathrm{NaCl}$, $\mathrm{LiCl}, \mathrm{KCl}, \mathrm{CaCl}_{2}$ and $\mathrm{AlCl}_{3}$ ) was measured in a concentration range $0.1-0.5 \mathrm{~mol} \mathrm{dm}^{-3}$. Although no significant alterations in the swelling degree can be found for the different 1:1 electrolytes, when the cation charge of unsymmetrical electrolytes increases, the gel swells in a significant way. This swelling process is enhanced by the presence of calyx[4]pyrrole. The effect of alkaline hydrolysis of polaycrylamide-based hydrogels was also studied. The hydrolysed hydrogels collapse in the presence of the electrolytes; this behavior is dependent on the hydrolysis degree, electrolyte charge and calyx[4]pyrrole presence and concentration; the latter leads to polyacrylamide with tailor-made properties.
\end{abstract}

(c) 2006 Elsevier Ltd. All rights reserved.

Keywords: Hydrogels; Polyacrylamide; Calyx[4]pyrrole; Swelling degree; Hydrolysis

\section{Introduction}

Porphyrinogens, also known as calix[4]pyrroles, are excellent chelating agents for binding anions [1-5] and neutral molecules [6], host materials for inclusion crystals [7] and also compounds for metal complexation [8-11].

\footnotetext{
* Corresponding author. Tel.: +351 239854459; fax: +351 239827703.

E-mail address: avalente@ci.uc.pt (A.J.M. Valente).
}

The past three decades have seen a growing interest in the use of neutral chelating agents to achieve ion permselectivity in membranes [12,13], which can be used for separation processes [14], or as sensing components in ion-selective electrodes [15].

Much work has been done incorporating neutral chelating agents, such as crown-ethers in hydrogels with low water content, in particular by physical immobilisation of crown-ethers in the water-swollen polymers [16].

Modified polyacrylamide hydrogels have also been developed to remove metallic ions (e.g., $\mathrm{Cu}^{2+}$ ) 


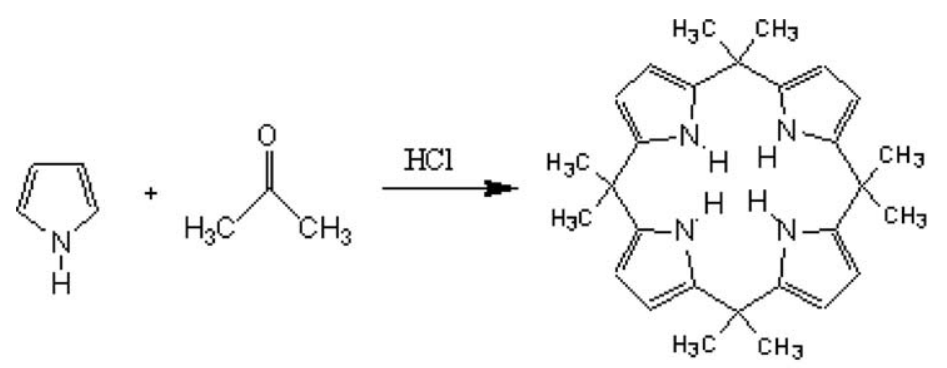

(1)

$5,5,10,10,15,15,20,20$ - Odamethyt porp hyrinogen

Scheme 1.

from wastewater [17]. Although some of those polymers have shown high ionic retention efficiency, the permeation of different fluids is quite low.

The synthesis (Scheme 1) and characterization of polyacrylamide membranes containing calyx[4]pyrrole (1) is reported. The consequences of the macrocycle inclusion in polyacrylamide membranes are shown in terms of swelling degree and calorimetric analysis. The effect of the macrocycle inclusion and alkaline hydrolysis in polyacrylamide membranes is shown in terms of differential scanning calorimetry (DSC), Fourier transformed infrared spectroscopy (FTIR), and swelling degree.

The reported results might help in finding new applications of these modified gels in the wastewater treatment (including recycling and recovering).

\section{Experimental section}

\subsection{Reactants}

Acrylamide (AAm), N, N'-methylene-bis-acrylamide (MBAAm) (used as crosslinker) and sodium persulfate (used as initiator) were purchased from Riedel-de-Haen.

Sodium chloride (Riedel-de Häen, Spain), calcium chloride (E. Merck, Darmstadt, Germany) and aluminium chloride (E. Merck, Darmstadt, Germany) were of pro analysis quality and were used without further purification. The electrolyte solutions were prepared after drying the salts until constant weight, and using bi-distilled water.

\subsection{Synthesis of meso-octamethyl-porphyrinogen (1)}

In a $100 \mathrm{~mL}$ round-bottom flask (equipped with a magnetic stirrer and a condenser) with $1 \mathrm{~mL}$ $(15 \mathrm{mmol})$ of pyrrole and $5 \mathrm{~mL}$ of acetone, five drops of concentrated $\mathrm{HCl}$ were added in a rapid sequence. The colourless solution turns to brown and shows a moderate effervescence that is followed by a rapid precipitation of calix[4]pyrrole, as a white solid. Calix[4]pyrrole is filtered, washed with $15 \mathrm{~mL}$ of cold acetone and dried at $40{ }^{\circ} \mathrm{C}$ for $30 \mathrm{~min}$ with a $70 \%$ yield. Infrared spectrum $(\mathrm{KBr}):\left(3440 \mathrm{~cm}^{-1}\right.$, $\mathrm{N}-\mathrm{H}$, strong) $\left(3104 \mathrm{~cm}^{-1}, \mathrm{C}-\mathrm{H}_{\text {aromatic }}\right.$, medium) (2886-2899 $\mathrm{cm}^{-1}, \mathrm{C}-\mathrm{H}_{\text {aliphatic }}$, strong) $\left(1578 \mathrm{~cm}^{-1}\right.$, $\mathrm{C}=\mathrm{C}_{\text {aromatic }}$, strong). ${ }^{1} \mathrm{H}$ NMR $\left(250 \mathrm{MHz}, \mathrm{CDCl}_{3} /\right.$ TMS $):(\delta 1.5, \mathrm{~s}, 6 \mathrm{H})(\delta 5.8, d=2.5 \mathrm{~Hz}, 2 \mathrm{H})(\delta 7.0$, broad s, $1 \mathrm{H}) .{ }^{13} \mathrm{C} \mathrm{NMR}\left(500 \mathrm{MHz}\right.$, in $\left.\mathrm{CDCl}_{3}\right)$ signals: $29.07 ; 35.16 ; 102.81 ; 138.4$. Mass spectra, $\mathrm{ESI}^{+}$, direct probe (dichloromethane, 5\% trifluoroacetic acid): $(\mathrm{M}+\mathrm{H})^{+}$at $m / z 429,100 \% ; m / z 413$, $15 \%, m / z 334,7 \%, m / z 275,16 \%$. Elemental analysis: N: $12.9 \%, \mathrm{C}: 77.19 \%, \mathrm{H}: 8.24 \%$; expected: N: $13.07 \%$, C: $78.46 \%$, H: $8.47 \%$.

\subsection{Polyacrylamide membranes}

\subsubsection{PAAm membranes preparation}

Pre-gel homogeneous aqueous solutions of AAm $(2.5 \mathrm{M})$, with $5 \%(\mathrm{~mol} / \mathrm{mol})$ of MBAAm and $1 \%$ of initiator were prepared. The hydrogel membranes were prepared by free radical polymerisation of the pre-gel solution [18] inside two glass sheets separated by plastic rubber gasket. Spring clips were used to hold the glass sheets together. After this procedure, the mould was placed in an oven at $50{ }^{\circ} \mathrm{C}$ for $2 \mathrm{~h}$. The hydrogel membrane was then removed from the mould, placed between two PVC sheets, and stored inside an exsiccator at nearly $100 \%$ humidity.

\subsubsection{PAAm/CD membranes preparation}

Three different gels were prepared by free radical polymerisation of the pre-gel solution obtained by mixing the above mentioned AAm solution, con- 
taining MBAAm and initiator, with a $\mathrm{CP}$ solution (previously prepared, $0.254 \mathrm{mM}$ in DMF), in different mixing ratios: 100/0; 90/10; 80/20 (v/v); PA-0, $[\mathrm{CP}]=0 \%(\mathrm{v} / \mathrm{v}) ; \mathrm{PA}-7,[\mathrm{CP}]=10 \%(\mathrm{v} / \mathrm{v})$; and PA14 with $20 \%(\mathrm{v} / \mathrm{v})$ of $\mathrm{CP}$.

The obtained membranes contain $0 \% \mathrm{CP}, 7 \%$ $(\mathrm{mg} / \mathrm{g}) \mathrm{CP}$ and $14 \%(\mathrm{mg} / \mathrm{g}) \mathrm{CP}$, respectively, relative to PAAm, and will be symbolised as PA-0, PA-7 and PA-14.

The membranes produced were clear, indicating that no phase separation had occurred between the polymer and calyx[4]pyrrole during the polymerisation process. On hydration, the membranes remained transparent, and this is consistent with the membranes retaining their homogeneity in the water-swollen state [16].

\subsection{Hydrolysis of hydrogel membranes}

The hydrolysis of membranes PA-0 and PA-14 was carried out under an alkaline medium at $25^{\circ} \mathrm{C}$. The sodium hydroxide concentration was maintained at $0.1 \mathrm{~mol} \mathrm{dm}^{-3}$ (gels PA-0a and PA14a) and $1.0 \mathrm{~mol} \mathrm{dm}^{-3}$ (gels PA-0b and PA-14b), and the reaction took up to $1 \mathrm{~h}$.

The membrane was then washed with a continuously flux of fresh water until all $\mathrm{NaOH}$ had been desorbed.

\subsection{Hydrogels swelling equilibrium in water and salts solutions}

The water swelling degree, $Q_{\text {sw }}$, of a hydrogel membrane, can be calculated from the weight of hydrated $\left(M_{\mathrm{gw}}\right)$ and dried (xerogel), $M_{x}$, samples, from $Q_{\mathrm{sw}}=M_{\mathrm{gw}} / M_{x}$. The total weight of hydrated sample was obtained after three weeks in equilibrium with water, and the weight of water in hydrated sample was obtained taking into account the mass of xerogel, calculated considering a total conversion of reagents in the polymerisation process [19].

The salt solution equilibrium swelling degree $Q_{\mathrm{ss}}$ $\left(=M_{\mathrm{gs}} / M_{x}\right)$ can be similarly determined from the weight of a membrane (swollen gel) after being in equilibrium with a salt solution, $M_{\mathrm{gs}}$. Each experiment was repeated at least three times and they were carried out at $25^{\circ} \mathrm{C}$.

\subsection{Polymer characterization}

Differential scanning calorimetry (DSC) measurements obtained from a modulated DSC instru- ment Q100 (TA Instruments, USA), equipped with a cooling unit (temperature range -180 to $725^{\circ} \mathrm{C}$ ). Calorimetric curves were obtained between $-50{ }^{\circ} \mathrm{C}$ and $220^{\circ} \mathrm{C}$ with a scan rate of $5^{\circ} \mathrm{C} / \mathrm{min}$. Sample weights of about $2.2 \mathrm{mg}$ were used and dry nitrogen was used as the purge gas.

The incorporation of $\mathrm{CP}$ into PAAm and the effect of hydrolysis in hydrogels membranes were characterized by their infrared spectra using a Vector 22/N, FTIR BRUKER spectrometer. Membranes were kept $24 \mathrm{~h}$ at $28{ }^{\circ} \mathrm{C}$ and at low pressure before analysis.

\section{Results and discussion}

\subsection{Polymer characterization}

Fig. 1 shows DSC thermograms of PAAm without (PA-0) and with 7 and 14 (mg/g) \% of CP, PA7 and PA-14, respectively. Thermograms of PA-7 and PA-14 (Fig. 1, curves b and c) show an endothermic variation after $140{ }^{\circ} \mathrm{C}$, which is due to glass transitions, $T_{\mathrm{g}}$, found at 173 and $154^{\circ} \mathrm{C}$, respectively. However, these thermograms are very different of that of pure PAAm (Fig. 1a), where no $T_{\mathrm{g}}$ is observed in the considered interval of temperatures. In fact, this is in agreement with literature where a $T_{\mathrm{g}}$ at $188^{\circ} \mathrm{C}$ is reported [20]. From this analysis, two major conclusions may be taken: alterations in thermograms confirm the modification of the hydrogel structure by incorporation of the calyx[4]pyrrole; and calyx[4]pyrrole acts as plasticizer decreasing the gel $T_{\mathrm{g}}$.

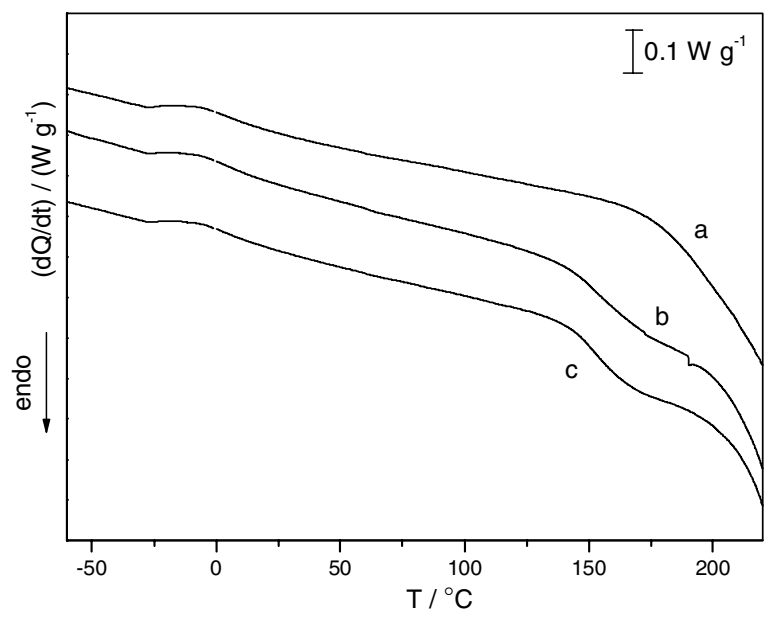

Fig. 1. DSC curves for heating runs performed on (a) PA-0, (b) PA-7 and (c) PA-14 at $5{ }^{\circ} \mathrm{C} / \mathrm{min}$. 
Due to the small amounts of calyx[4]pyrrole used to prepare polyacrylamide gels, an identification of specific peaks of $\mathrm{CP}$, resulting from such incorporation, becomes a difficult task. Fig. 2 shows FTIR spectra of PAAm without (PA-0) and with 14\% CP (PA-14) in powder forms. FTIR spectrum of PA-0 shows two specific peaks at $3180 \mathrm{~cm}^{-1}$ (bonded $-\mathrm{NH}_{2}$ stretching) and $1636 \mathrm{~cm}^{-1}$ due to $-\mathrm{NH}_{2}$ bending. Both peaks also appear to PA-14

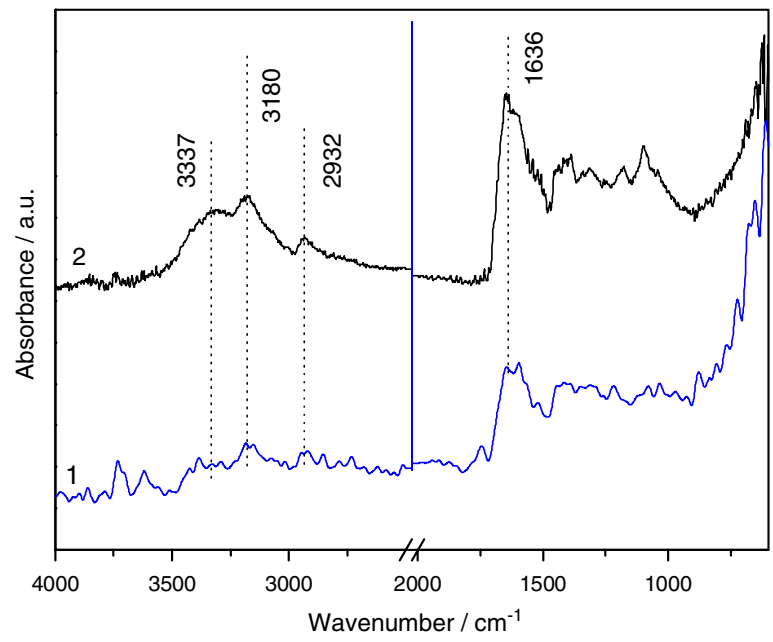

Fig. 2. Infrared spectra of (1) PA-0 and (2) PA-14. sample but with broader peaks; the first at $3337 \mathrm{~cm}^{-1}$ as due to $\mathrm{NH}$ groups of pyrrole and the second (well defined to PA-14) probably due to the overlapping of the specific characteristic bands of $\mathrm{C}=\mathrm{C}_{\text {aromatic }}$ vibrational mode. Besides such alterations, it is worthwhile to note an appearance of a peak at $2932 \mathrm{~cm}^{-1}$ due to $\mathrm{CH}$ aliphatic groups of $\mathrm{CP}$.

The effect of alkaline hydrolysis on the structural properties of polyacrylamide and CP-containing polyacrylamide (in membranes) was followed by FTIR and is shown in Figs. 3 and 4, respectively. It is possible to observe that the characteristic absorption bands of PAAm $\left(3336 \mathrm{~cm}^{-1}\right.$ : free- $\mathrm{NH}_{2}$, stretching; $3190 \mathrm{~cm}^{-1}$ : bonded- $\mathrm{NH}_{2}$ stretching; $1650 \mathrm{~cm}^{-1}:-\mathrm{C}=\mathrm{O}$ stretching; $1602 \mathrm{~cm}^{-1}:-\mathrm{NH}_{2}$ bending; and $1471 \mathrm{~cm}^{-1}$ : $\mathrm{C}-\mathrm{N}$ stretching) [21,22] are present in all spectra.

By comparing the effect of $\mathrm{NaOH}$ treatment in polyacrylamide gels without and with $\mathrm{CP}$ (Figs. 3 and 4 , respectively) it is possible to conclude that: (a) the intensity of the band at $3190 \mathrm{~cm}^{-1}$ (assigned to $\mathrm{N}-\mathrm{H}$ bond) [23] decreases when $\mathrm{NaOH}$ concentration increases, for CP-containing gels (see arrow I in Figs. 3 and 4); and (b) the intensity of bands at $1650 \mathrm{~cm}^{-1}$ (assigned to $\mathrm{C}=\mathrm{O}$ bond from amides) and at $1602 \mathrm{~cm}^{-1}$ (assigned to deformation vibra-

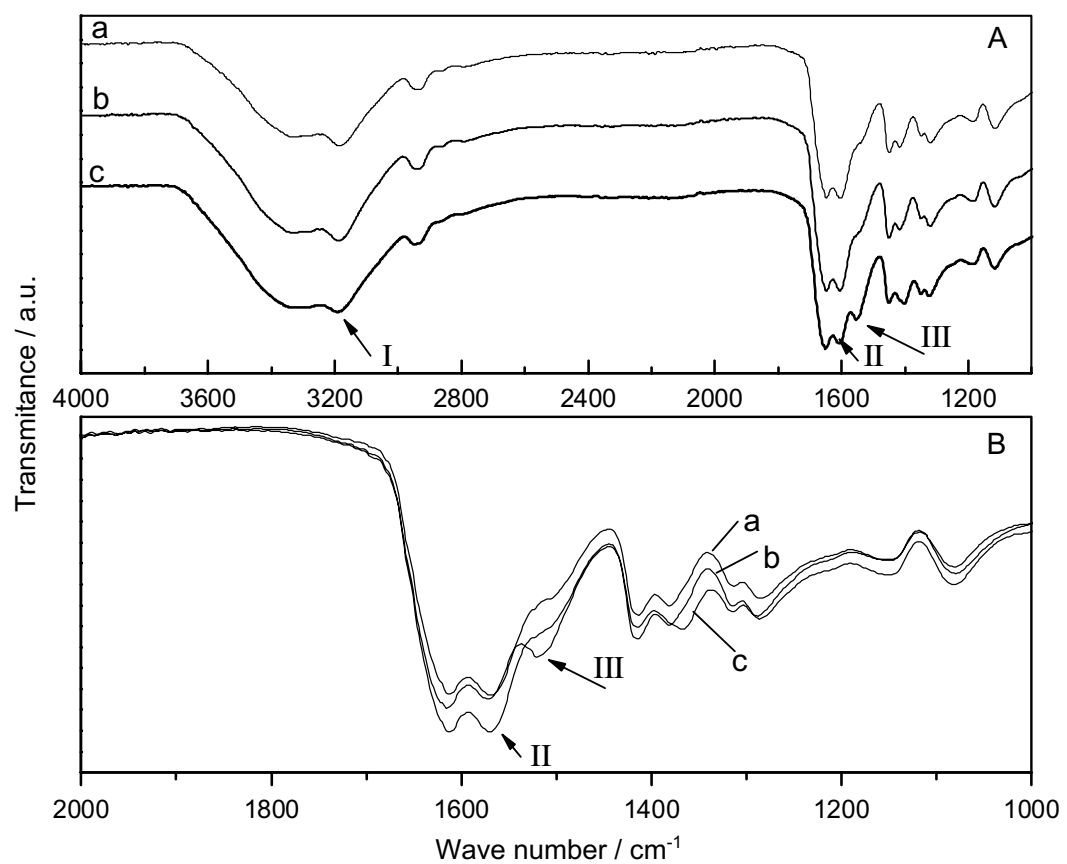

Fig. 3. A. FTIR spectra of polyacrylamide (a) and modified polyacrylamide with $0.1 \mathrm{M} \mathrm{NaOH} \mathrm{(b)} \mathrm{and} 1.0 \mathrm{M} \mathrm{NaOH} \mathrm{(c).} \mathrm{I:} 3190 \mathrm{~cm}^{-1}$, II: $1602 \mathrm{~cm}^{-1}$, III: $1553 \mathrm{~cm}^{-1}$. B. Inset figure: FTIR spectra overlapping to clarify the hydrolysis effect. 


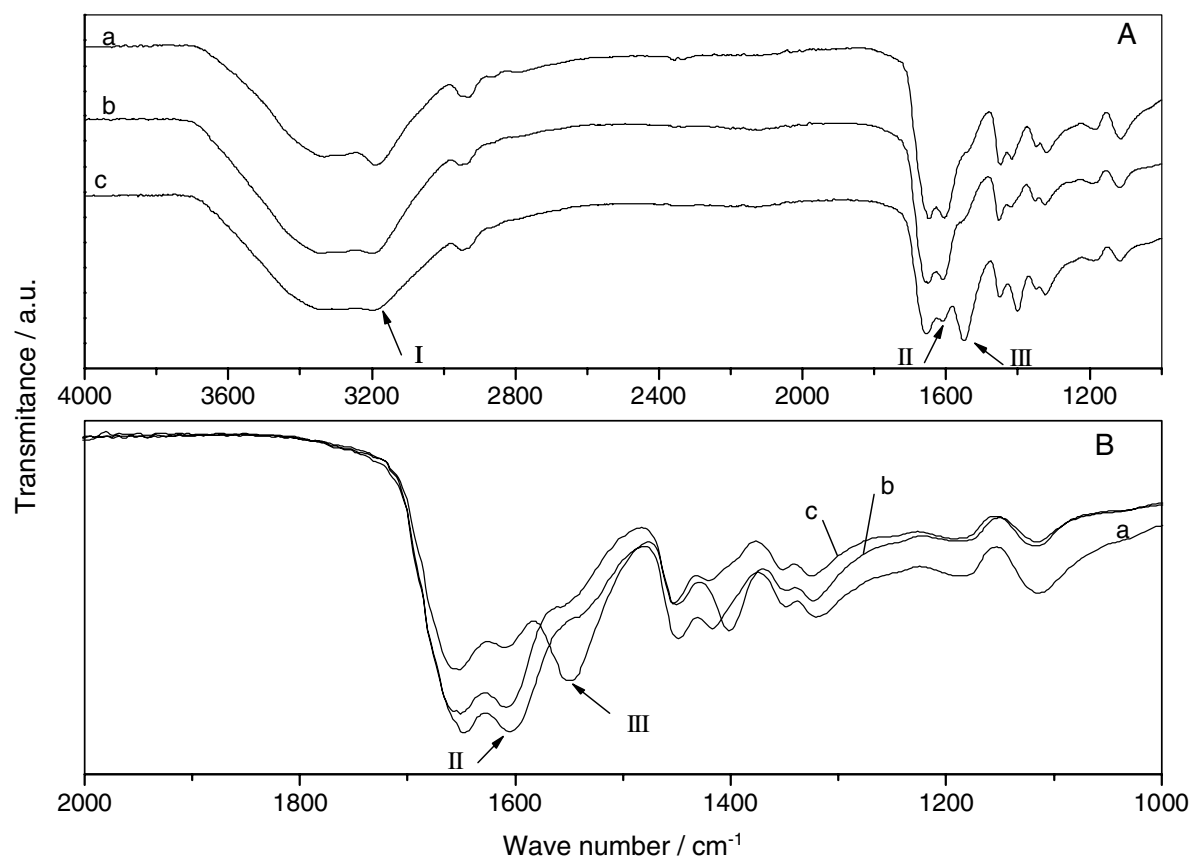

Fig. 4. A. FTIR spectra of polyacrylamide-containing calyx[4]pyrrole (a, PA-14), and modified polyacrylamide-containing calyx[4]pyrrole hydrolysed with $0.1 \mathrm{M} \mathrm{NaOH}$ (b, PA-14a) and $1.0 \mathrm{M} \mathrm{NaOH}$ (c, PA-14b). I: $3190 \mathrm{~cm}^{-1}$, II: $1602 \mathrm{~cm}^{-1}$, III: $1553 \mathrm{~cm}^{-1}$. B. inset figure: FTIR spectra overlapping to clarify the hydrolysis effect.

tions of $\mathrm{NH}_{2}$ bonds - see arrow II in Figs. 3 and 4) decreases drastically when $\mathrm{NaOH}$ and $\mathrm{CP}$ concentrations increase. This intensity decrease is accompanied by an increasing of the absorption at $1550 \mathrm{~cm}^{-1}$ (assigned to the carboxylate groups). In fact, shoulders at $1550 \mathrm{~cm}^{-1}$, which can be found in the untreated membrane spectrum (a spectra in Figs. 3 and 4), became more pronounced when the membranes were treated with a $0.1 \mathrm{M} \mathrm{NaOH}$ solution and they became a sharp band when the membrane was treated with a more concentrated $(1.0 \mathrm{M})$ $\mathrm{NaOH}$ solution [24]. This means that the presence of $\mathrm{CP}$ in the membranes makes the amide groups from PAAm more accessible to the $\mathrm{NaOH}$ attack, due to the high dimensions of CP cycles that determine the moving of the PAAm chains. So, increasing $\mathrm{NaOH}$ concentration will determine the increase of the PAAm hydrolysis, shown by the absorption bands of the carboxylate group $\left(1550 \mathrm{~cm}^{-1}\right)$.

The intensity of the absorption at $1417 \mathrm{~cm}^{-1}$, which can be assigned to the carboxylate group [25], increases with increasing the $\mathrm{NaOH}$ concentration, showing the PAAm hydrolysis. The increase of this absorption is larger in the presence of $\mathrm{CP}$ (Fig. 4) compared to that of the membrane without CP (Fig. 3).
The influence of the $\mathrm{NaOH}$ concentration on the hydrolysis degree of PAAm, in the absence and the presence of $\mathrm{CP}$, can be observed in Figs. 3 and 4, respectively. The increase of the hydrolysis degree of PAAm in the presence of $\mathrm{CP}$ and with the increase of the $\mathrm{NaOH}$ solution concentration could be seen when we compare the ratio between the absorption at $1553 \mathrm{~cm}^{-1}$ (carboxylate group) and $1602 \mathrm{~cm}^{-1}$ of the amide group (Table 1).

Results in Table 1 show that no significant hydrolysis occurs when $0.1 \mathrm{M} \mathrm{NaOH}$ is used, and, at more caustic conditions, $\mathrm{CP}$ has a non-neglected influence on the hydrolysis process. In fact, it can be expected that in the presence of CP the free space between polymer molecular chains increases, and consequently a higher access of $\mathrm{NaOH}$ inside membrane occurs. This may explain an increase of $57 \%$ of $I_{1553} / I_{1602}$ in the presence of CP when compared with the homologue without CP.

This hypothesis is supported by the water absorption analysis. The swelling degree of PA- 0 , PA-7 and PA-14 slightly increases: $6.27( \pm 0.03)$, $6.47( \pm 0.02)$ and $7.05( \pm 0.02)$, respectively. When PA-0 and PA-14 gels suffer alkaline hydrolysis in a small extension $(\mathrm{NaOH} 0.1 \mathrm{M})$, swelling degree also increases to $6.38( \pm 0.03)$ and $7.15( \pm 0.03)$. A more 


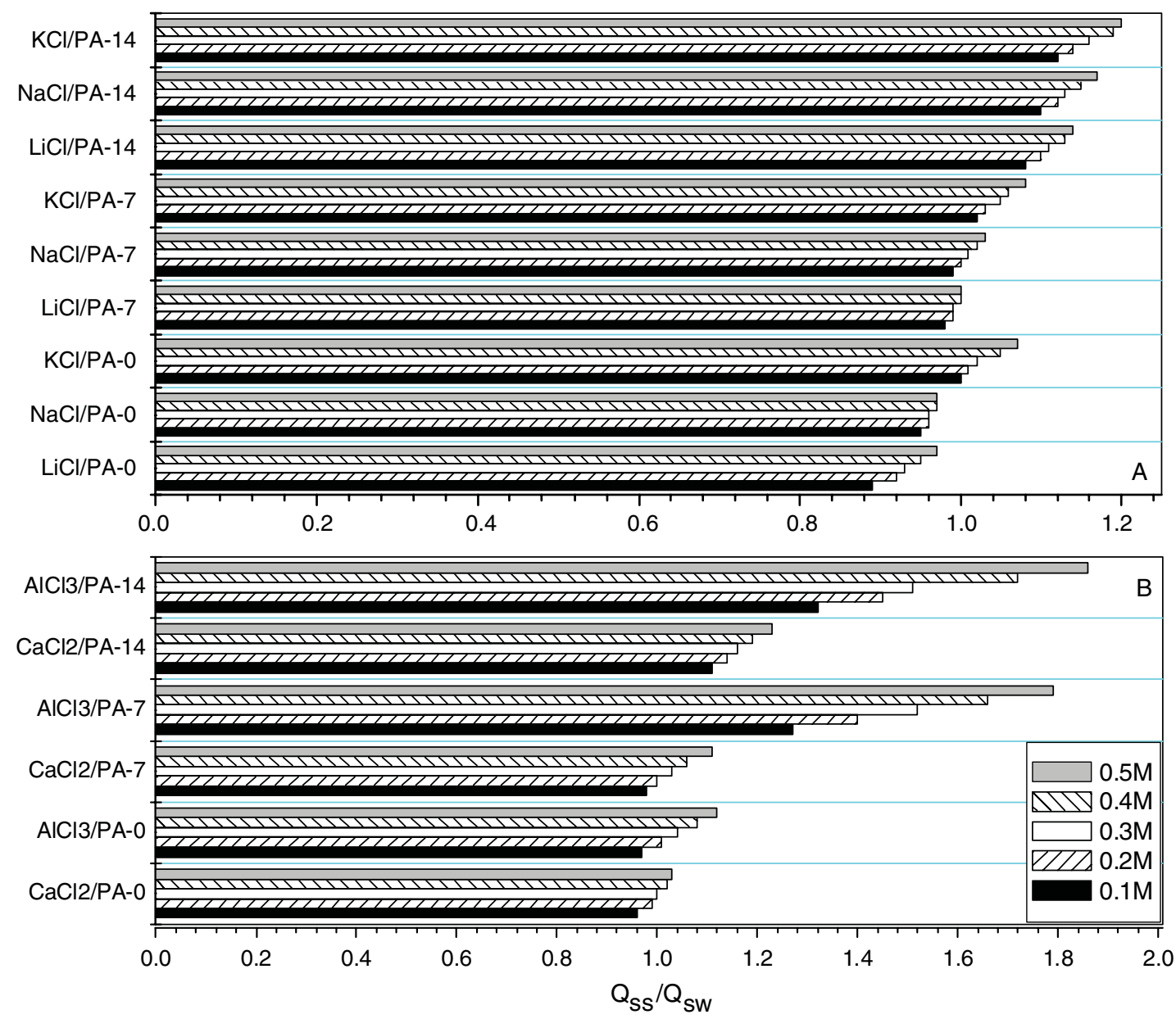

Fig. 5. Normalized swelling degree, $Q_{\mathrm{ss}} / Q_{\mathrm{sw}}$, of polyacrylamides in aqueous solutions of (A) symmetrical univalent ( $\mathrm{LiCl}, \mathrm{NaCl}$ and $\mathrm{KCl}$ ) and (B) of unsymmetrical $\left(\mathrm{CaCl}_{2}\right.$ and $\left.\mathrm{AlCl}_{3}\right)$ electrolytes, at $25^{\circ} \mathrm{C}$. The error bars are not drawn to avoid confusion but the maximum error in the swelling degree calculation is $5 \%$.

Table 1

Effect of sodium hydroxide and calyx[4]pyrrole concentration on the hydrolysis of polyacrylamide and on the water swelling of membranes

\begin{tabular}{|c|c|c|c|c|c|}
\hline Sample & {$[\mathrm{NaOH}] / \mathrm{M}$} & $I_{1602}$ & $I_{1553}$ & $I_{1553} / I_{1602}$ & $Q_{\text {sw }}$ \\
\hline $\begin{array}{l}\text { PAAm } \\
\text { (PA-0) }\end{array}$ & 0 & 0.492 & 0.236 & 0.479 & $6.27( \pm 0.03)$ \\
\hline $\begin{array}{l}\text { PAAm } \\
\text { (PA-0a) }\end{array}$ & 0.1 & 0.551 & 0.277 & 0.502 & $6.38( \pm 0.02)$ \\
\hline $\begin{array}{l}\text { PAAm } \\
\qquad \text { (PA-0b) }\end{array}$ & 1.0 & 0.467 & 0.327 & 0.700 & $9.24( \pm 0.02)$ \\
\hline $\begin{array}{l}\text { PAAm + CP } \\
(\text { PA-14) }\end{array}$ & 0 & 0.518 & 0.225 & 0.434 & $6.47( \pm 0.01)$ \\
\hline $\begin{array}{c}\text { PAAm + CP } \\
\text { (PA-14a) }\end{array}$ & 0.1 & 0.483 & 0.214 & 0.443 & $7.15( \pm 0.02)$ \\
\hline $\begin{array}{c}\text { PAAm + CP } \\
\text { (PA-14b) }\end{array}$ & 1.0 & 0.325 & 0.357 & 1.100 & $9.94( \pm 0.02)$ \\
\hline
\end{tabular}

dramatic change in swelling degree is found when gel membranes are previously immersed in $\mathrm{NaOH}$
1.0 M. Under these circumstances, the swelling degree for both gels, PA-0 and PA-14, increases to $9.24( \pm 0.04)$ and $9.94( \pm 0.05)$, respectively.

The above results deserve the following comments: (a) $Q_{\text {sw }}$ increases with $\mathrm{CP}$ content inside gel, following a linear trend; (b) $Q_{\text {sw }}$ of PA-0a and PA-14a are slightly higher than those found to PA-0 and PA-14, following the same displacement found by FTIR analysis; and (c) gels PA-0b and PA-14b suffer a dramatic increase of $Q_{\text {sw }}$.

In the presence of $\mathrm{NaOH}$, the sorption is accompanied by an alkaline hydrolysis process and consequently by the formation of an anionic gel. The swelling behavior can be explained on the basis of repulsions between fixed charged groups on the gel. These repulsions are long-range and the gel expands to minimize the repulsive free energy [26]. 
From the swelling degree and FTIR data, it is also observed that the hydrolysis displacement in gel PA-14c (ca. 150\%, taking as reference the gel PA-14) is not accompanied in a such large extension by the swelling degree (ca. $40 \%$ ). In the case of alkaline hydrolysis, a small alteration in the water swelling degree occurs (see Table 1). However, it is worth noting that the swelling of the hydrogel is enhanced in the CP-incorporated PAAm gel clearly suggesting that $\mathrm{CP}$ acts as plasticizer; in this way, $\mathrm{CP}$ will contribute to the increase of available amide groups that suffer hydrolysis, which is in agreement with DSC analysis.

\subsection{Effect of non-associated electrolytes on the swelling degree of $P A A m / C P$ membranes}

The effect of different electrolytes in the swelling degree of polyacrylamides with and without $\mathrm{CP}$ incorporation was analysed. Fig. 5(A) shows the dependence of normalized swelling degree $\left(\mathrm{NSD}=Q_{\text {ss }} / Q_{\text {sw }}\right)$ of gels on different $\mathrm{LiCl}, \mathrm{NaCl}$ and $\mathrm{KCl}$ aqueous solutions. Although no significant alterations in $Q_{\mathrm{ss}}$ concerning different electrolytes can be found, in a general way, the swelling degree increases with electrolyte concentration. Comparing the results in PA-0, it is possible to observe that $Q_{\mathrm{ss}}$ increases in the following order: $\mathrm{LiCl}<\mathrm{NaCl}<$ $\mathrm{KCl}$. These results suggest that, at $0.1 \mathrm{M}$, the gel slightly shrinks in the presence of a high cation charge density and slightly swells in the presence of the highest crystallographic cation radius. In fact, the structurant effect of the cations in the water structure is as follows: $\mathrm{Li}^{+}>\mathrm{Na}^{+}>\mathrm{K}^{+}$[27]. This effect can also explain the highest solubility of $\mathrm{LiCl}$ (highest dependence of $Q_{\text {ss }}$ on concentration) in PA0 when compared with other 1:1 electrolytes [28].

In the presence of $\mathrm{CP}$ the swelling degree of PA-7 and PA-14 increases until 20\% (PA-14/KCl). This shows that interactions between cation and $\mathrm{CP}$ occur and, consequently, the counter-ion concentration inside gel also increases causing the gel expansion. Cation interaction energy minimization using the semi-empirical method MM2 (Cambridge Software, 2001, v. 7.0.0) shows that $\mathrm{K}^{+} / \mathrm{CP}$ and $\mathrm{Li}^{+} /$ $\mathrm{CP}$ have an energy of $-45.23 \mathrm{kcal} / \mathrm{mol}$ and $-65.45 \mathrm{kcal} / \mathrm{mol}$, respectively. The energy in the $\mathrm{Na}^{+}$system is between those of the $\mathrm{K}^{+}$and $\mathrm{Li}^{+}$systems. These results, however, do not take into account the presence of water; in these circumstances, these values may suffer significant alterations. A possible explanation may be found on the basis of the hydration radius analysis; once the hydration radius of $\mathrm{K}^{+}$is smaller than that of $\mathrm{Li}^{+}$, we may expect a greater interaction on hydrated $\mathrm{K}^{+} / \mathrm{CP}$ and, consequently, in this system the ionic strength inside the matrix increases due to an increase of free counter-ions, and therefore $Q_{\text {ss }}$ is the highest of all the 1:1 electrolytes. Concluding, the presence of CP has influence on the degree of swelling of PAAm but these polymers are not selective to 1:1 symmetrical non-associated electrolytes.

The effect of cationic charge on the NSD of different polyacrylamides is shown in Fig. 5(B). The charge effect is predominant on the swelling process. Comparing the effect of the electrolyte symmetry, the structurant effect of $\mathrm{Ca}^{2+}$ is much higher than in the case of univalent cations. Consequently, a collapse of the PA- 0 at low concentrations occurs; when the concentration increases the ionic strength, not only it cannot be neglected, but it plays the main role on the process (that is, the gel swells). However the largest charge effect (an increase of $Q_{\text {ss }}$ up to $50 \%$ ) occurs in the presence of $\mathrm{CP}$. With an increase of the $\mathrm{Ca}^{2+}-\mathrm{CP}$ and $\mathrm{Al}^{3+}-\mathrm{CP}$ associations, the internal salt concentration rises, as well as the concentration of the counter-ions, the difference between the internal and external ion concentrations increases, and the gel swells due to an increase in osmotic pressure [29]. It is also important to point out that the effect of $\mathrm{CP}$ incorporation is lower when the cationic charge increases from 2 to 3.

\subsection{Effect of electrolytes on the swelling degree of hydrolysed PAAm/CP membranes}

The swelling properties of PAAm/CP gels are influenced by $\mathrm{NaOH}$ sorption and this may be resumed in the following way: with the base-catalyzed amide hydrolysis, a formation of carboxylates and ammonia occurs. In the present case, ammonia can be leached out during the hydrogel washing process. The functionalization of amide to carboxylate groups is of practical importance, increasing the retention capacity by hydrogels to cations as will be analysed.

Taking into consideration the experimental results shown in previous sections, the effect of cationic charge was checked for $\mathrm{NaCl}, \mathrm{CaCl}_{2}$ and $\mathrm{AlCl}_{3}$ in the concentration range $0.01-0.1 \mathrm{M}$ (Fig. 6). Once the sorption of these electrolytes leads to a shrinkage of hydrolysed gels, the results will be discussed in terms of the normalized collapse degree, given by the $Q_{\mathrm{sw}} / Q_{\mathrm{ss}}\left(=M_{\mathrm{gw}} / M_{\mathrm{gs}}\right)$ ratio; in 


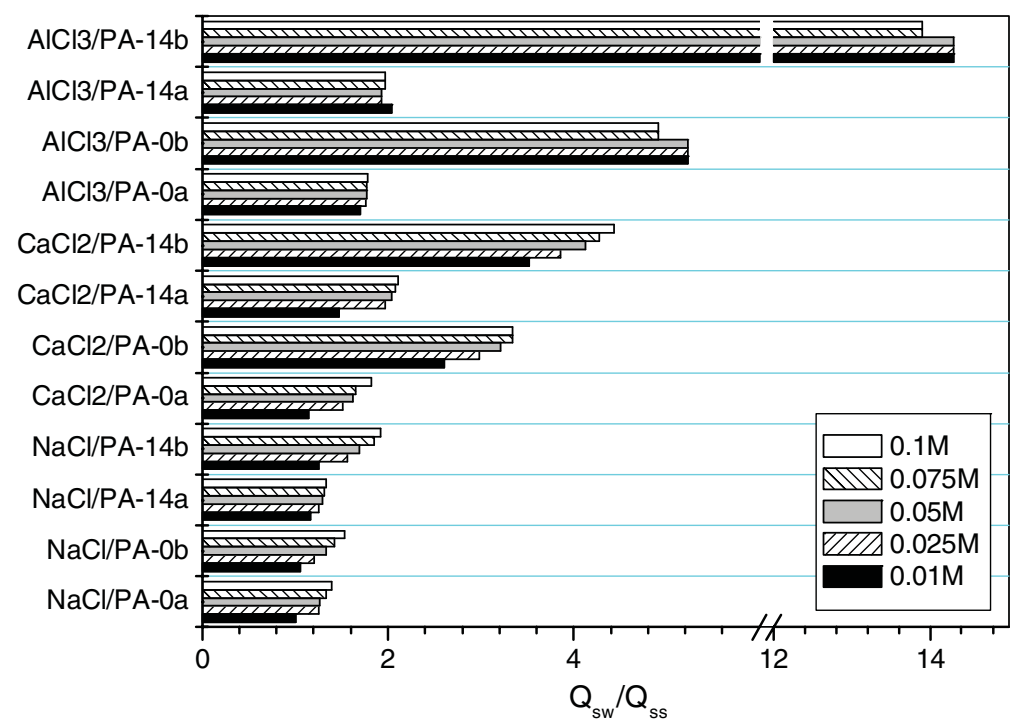

Fig. 6. Normalized collapse degree of hydrolysed hydrogels in equilibrium with different electrolyte $\left.\left(\mathrm{NaCl}_{2} \mathrm{CaCl}{ }_{2} \text { and } \mathrm{AlCl}\right)_{3}\right)$ aqueous solutions. The error bars are not drawn to avoid confusion but the maximum error in the swelling degree calculation is $5 \%$.

Table 2

Fitting parameters obtained from a linear regression of experimental data (Fig. 6) to Eq. (1)

\begin{tabular}{|c|c|c|c|c|c|c|}
\hline & \multicolumn{2}{|l|}{$\mathrm{NaCl}$} & \multicolumn{2}{|l|}{$\mathrm{CaCl}_{2}$} & \multicolumn{2}{|l|}{$\mathrm{AlCl}_{3}$} \\
\hline & $m / M^{-1}$ & $\left(Q_{\mathrm{sw}} / Q_{\mathrm{ss}}\right)_{0}$ & $m / M^{-1}$ & $\left(Q_{\mathrm{sw}} / Q_{\mathrm{ss}}\right)_{0}$ & $m / M^{-1}$ & $\left(Q_{\mathrm{sw}} / Q_{\mathrm{ss}}\right)_{0}$ \\
\hline PA-0a & $2.1(0.3)$ & $1.18(0.02)$ & $4.1(0.8)$ & $1.39(0.05)$ & $0.2(0.1)$ & $1.8(0.1)$ \\
\hline PA-0b & $4.6(0.4)$ & $1.08(0.02)$ & $5.0(1.7)$ & $2.9(0.1)$ & ca. 0 & $5.1(1.2)$ \\
\hline PA-14a & $1.1(0.3)$ & $1.22(0.02)$ & $2.0(0.3)$ & $1.8(0.1)$ & $1.4(0.4)$ & $2.0(0.1)$ \\
\hline PA-14b & $5.2(0.6)$ & $1.42(0.04)$ & $7.8(1.1)$ & $4.0(0.5)$ & ca. 0 & $14.1(1.0)$ \\
\hline
\end{tabular}

The data inside brackets are standard deviations.

this way, we will maintain, as reference, the mass of gel in equilibrium with water.

Fig. 6 shows that, in the presence of electrolytes, shrinkage of hydrolysed gels occurs. The collapse of gels may be explained on the basis of a simple discussion [26]: at low ionic strength, the concentration of the bound charges (and accompanying co-ions) within gel exceeds the concentration of electrolyte in the external solution; a large swelling pressure causes the gel to expand, thereby lowering the concentration of the co-ions within the gel; and the collapse of gels can also be explained on the basis of repulsions between fixed charged groups on the gel. As ionic strength rises inside gel, repulsions are shielded and the gel collapses.

Analysis of experimental data shown in Fig. 6 suggests an increase of $Q_{\text {sw }} / Q_{\text {ss }}$ as a function of electrolyte concentration; such an increase, if we exclude the lowest electrolyte concentration, follows a linear trend. Table 2 presents the fitting parameters of the experimental data (Fig. 6) using
$\left(Q_{\mathrm{sw}} / Q_{\mathrm{ss}}\right)=\left(Q_{\mathrm{sw}} / Q_{\mathrm{ss}}\right)_{0}+m c$

where $m$ and $\left(Q_{\mathrm{sw}} / Q_{\mathrm{ss}}\right)_{0}$ are the gradient and the intercept of the regression line, respectively, and $c$ is the electrolyte concentration. The fitting parameters were obtained using Origin 6.0 software taking 95\% confidence limits.

From this analysis, the intercept $\left(\left(Q_{\mathrm{sw}} / Q_{\mathrm{ss}}\right)_{0}\right)$ gives information about the sensitivity of the gel to cationic valency, whilst the slope $m$ is a measure of the response of the gel to the alterations in electrolyte concentration.

Particular attention should be paid to values of $\left(Q_{\text {sw }} / Q_{\text {ss }}\right)_{0}$ obtained with different systems. This parameter gives indication of the magnitude of gel's collapse and, consequently, gives some indication about the effect of both electrolyte and CP incorporation upon hydrolysed hydrogels. Fig. 7 shows that the dependence of that parameter on cation charge, $Z$, follows a linear relationship. Therefore, for all gels, the ionic charge effect is the predominant fac- 

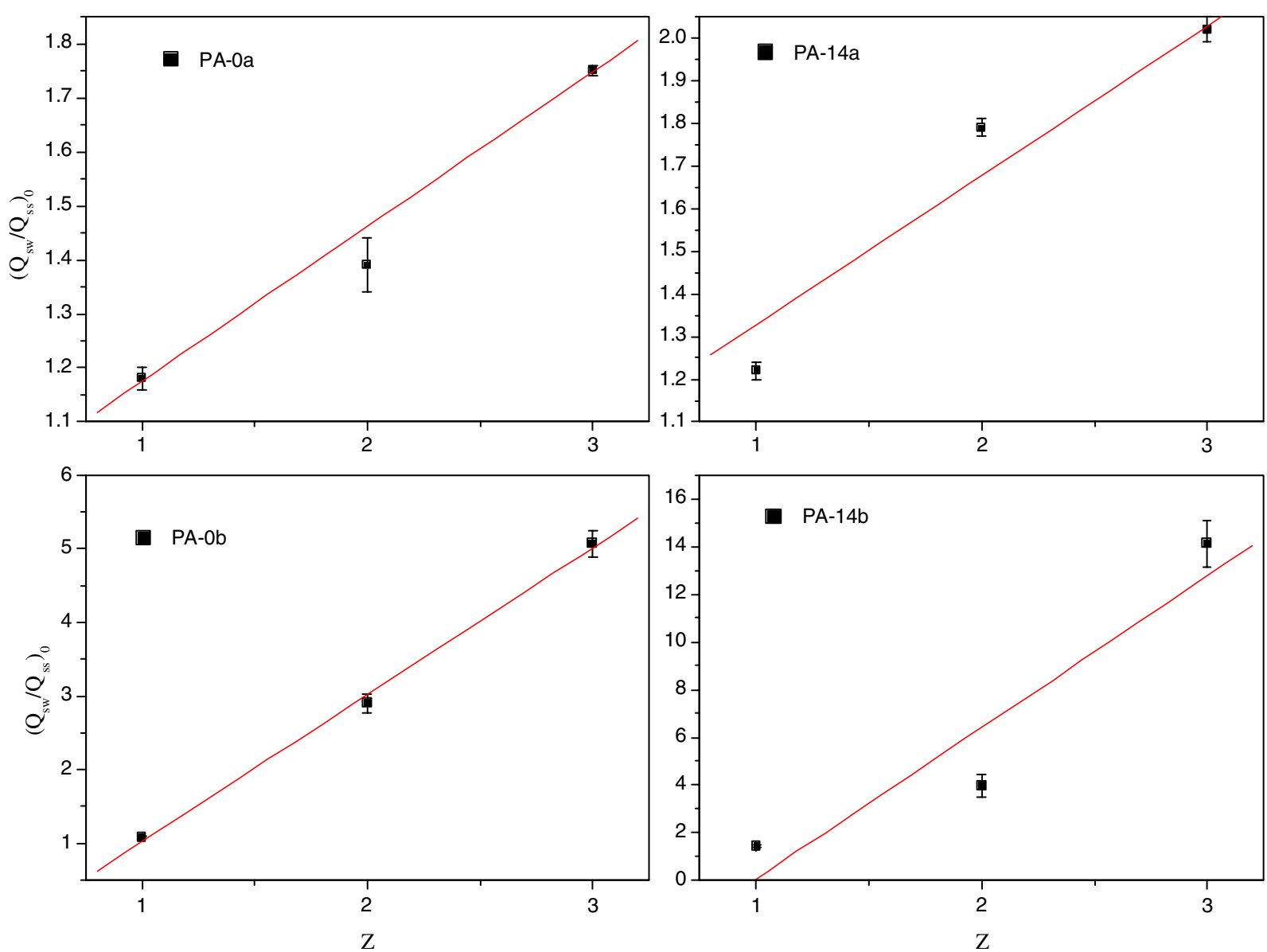

Fig. 7. Effect of cationic charge $(Z)$ on the intercept of Eq. (1), for different hydrolysed gels. The lines reproduces the fitting of the data points to a straight line.

tor on the shrinkage process. Other conclusions are: (a) $\mathrm{NaCl}$ is the less selective electrolyte towards the different gels; (b) the sorption of $\mathrm{AlCl}_{3}$ shows a high selectivity (PA-0a $\leqslant$ PA-14a $<$ PA- 0 b $\ll$ PA-14b) to the different gels under study; and (c) the presence of $\mathrm{CP}$ has always influence on the electrolyte sorption.

In the case of $\mathrm{NaCl}$, the electrolyte cation is the same than the hydrogel carboxylate counter-ion. The exchange $\mathrm{Na}_{\text {gel }}^{+}-\mathrm{Na}_{\mathrm{NaCl}}^{+}$does not occur and, if it occurs, does not significantly alter the properties of the whole gel matrix. The swelling degree of the gels is also dependent on $\mathrm{NaCl}$ concentration (see $m$ values in Table 2). Therefore, the collapse of the gel is mainly due to the screening effect on the anionic hydrogel groups.

When the cationic electrolyte charge increases to $+2\left(\mathrm{Ca}^{2+}\right)$, the magnitude of the collapse as well as the dependence on the $\mathrm{CaCl}_{2}$ concentration increases. The analysis of $\mathrm{AlCl}_{3}$ results deserves, however, further discussion: why is there a drastic collapse of the gels in the presence of aluminium ions and, simultaneously, the effect of $\mathrm{AlCl}_{3}$ concentration on $\left(Q_{\mathrm{sw}} / Q_{\mathrm{ss}}\right)$ is much lower ( $m$ are approx. zero to PA-0b and PA-14b) than in other electrolytes? The highest collapse which occurs in these systems cannot be justified only by a screening effect resulting from $\mathrm{AlCl}_{3}$ sorption. The exchange of $\mathrm{Na}^{+}$counter-ions by $\mathrm{Al}^{3+}$ is favoured by the ion charge and consequently it seems that $\mathrm{Al}^{3+}$ can act as a further "crosslinker", by bonding different anionic charged groups of the hydrogel; these two effects altogether will justify such collapse. Concerning the second point, an explanation comes as a consequence of the previous answer; the collapse of $\mathrm{Al}^{3+} /$ gel is accompanied by an increase of free counter-ions, which contribute to an increase of the swelling pressure inside the gel; the balance of these two facts will justify such $Q_{\mathrm{ss}}$ behavior. When the gel shows a highest degree of hydrolysis, the number of interactions between cation and anionic 
groups of PAAm increases and the effect of osmotic pressure is not so important.

Finally, it seems that $\mathrm{CP}$ has a clear influence on the electrolyte sorption as well as on the hydrolysis process. Independently of the interaction mechanism between electrolyte/CP and/or $\mathrm{CP} / \mathrm{gel}$, we may point out that a synergestic effect of calyx[4]pyrrole on the sorption of electrolytes can be reported; such synergism may be described not only by the possible ability to complex cations as well as to contribute to increase the number of amide groups that suffer hydrolysis; that is, $\mathrm{CP}$ can be suggested for producing tailored gels.

\section{Conclusions}

A new synthesis of a calyx[4]pyrrole is described. This synthesis procedure is quick, with high yield, and low cost. The swelling degree of the hydrogels based on PAAm, with and without $\mathrm{CP}$, in equilibrium with symmetrical and unsymmetrical electrolytes, with different cations, is dependent on the hydration radius (in symmetrical electrolytes) and on the ionic charge (in unsymmetrical salts). The effect of the cationic charge in the swelling degree of partially hydrolysed PAAm gels is also presented; retention of salts increases in hydrolysed gels, and could be observed by a collapse of the gels; such effect (collapse) increases with increasing of the cationic charge. This is explained on the basis of a selective exchange between $\mathrm{Na}^{+}$counter-ion of the hydrolysed gel with the external cation $\left(\mathrm{Na}^{+}, \mathrm{Ca}^{2+}\right.$ or $\left.\mathrm{Al}^{3+}\right)$. This interaction is accompanied by an increase of electrostatic bonds between cation and ionic gel structure when the cationic charge increases and, consequently, the gel collapse is higher in $\mathrm{Al}^{3+}$ systems.

The incorporation of CP in PAAm gels (with and without suffering hydrolysis) enhances the selectivity properties of gels for different electrolytes. The use of $\mathrm{CP}$ to prepare tailored gels is also an important outcome of the present study. This work allows the preparation of gels which can be useful to, e.g., anodizing companies, for salt retention and then for possible metallic recovery, or in the case where recovery is not economically acceptable, for drastically reducing the metallic-containing solid wastes.

\section{Acknowledgements}

We would like to thank Professor Dr. Christian Friedrich from Ludwigs-Albert University Frei- burg, Germany, for FTIR analysis. Financial support from POCTI/FCT/FEDER (AMB2004/ 55281, MAT2004/03827) and MEC/CRUP (Acções Integradas) is gratefully acknowledged.

\section{References}

[1] Gale PA, Sessler JL, Kral V, Lynch V. J Am Chem Soc 1996;118:5140.

[2] Sessler JL, Anzenbacher Jr P, Jursikova K, Miyaji H, Genge JW, Tvermoes NA, et al. Pure Appl Chem 1998;70: 2401.

[3] Jang Y-S, Kim H-J, Lee P-H, Lee C-H. Tetrahedron Lett 2000;41:2919.

[4] Nagarajan A, Ka J-W, Lee C-H. Tetrahedron 2001;57: 7323.

[5] Gale PA, Sessler JL, Allen WE, Tvermoes NA, Lynch V. Chem Commun 1997:665.

[6] Allen WE, Gale PA, Brown CT, Lynch V, Sessler JL. J Am Chem Soc 1996;118:12471.

[7] Furusho Y, Aida T. Chem Commun 1997:2205.

[8] Jacoby D, Floriani C, Chiesi-Villa A, Rizzoli C. Chem Commun 1991:220.

[9] Campazzi E, Solati E, Scopelliti R, Floriani C. Inorg Chem 1999;38:6240.

[10] Jacoby D, Floriani C, Chiesi-Villa A, Rizzoli C. J Am Chem Soc 1993;115:3595.

[11] De Angelis S, Solari E, Floriani C, Chiesi-Villa A, Rizzoli C. J Chem Soc Dalton Trans 1994:2467.

[12] Warshawsky A. Polymeric ligands in hydrometallurgy. In: Sherrington DC, Hodge O, editors. Synthesis and separations using functional polymers. Chichester: John Wiley; 1988. p. 325.

[13] Ciardelli E, Tsuchida E. In: Whorle D, editor. Macromolecular metal complexes. Berlin: Springer; 1996.

[14] Reusch CF, Cussler EL. AIChE J 1973;19:736.

[15] Koryta J, Stulik K. Ion selective electrodes. London: Cambridge University Press; 1984 [Chapter 7].

[16] Hamilton CJ, Murphy SM, Tighe BJ. Polymer 2000;41:3651.

[17] Kasgoz H, Ozgumus S, Orbay M. Polymer 2001;42:7497.

[18] Lobo VMM, Valente AJM, Polishchuk AYa, Geuskens G. J Mol Liquids 2001;94:179.

[19] Feng Y, Billon L, Grassl B, Khoukh A, François J. Polymer 2002;43:2055.

[20] Ribeiro-Silva MES, Machado JC, Mano V, Silva CG. J Polym Sci B: Polym Phys 2003;41:1493.

[21] Erbil C, Uyanik N. Polym Int 2001;50:792.

[22] Gupta B, Anjum N. J Appl Polym Sci 2003;90:3747.

[23] Jin L, Bai R. Langmuir 2002;18:9765.

[24] Muller G, Laine JP, Fenyo C. J Polym Sci: Polymer Chem Ed 1979;17:659.

[25] Kasgoz H, Ozgumus S, Orbay M. Polymer 2003;44:1785.

[26] Hooper HH, Baker JP, Blanch HW, Prausnitz JM. Macromolecules 1990;23:1096.

[27] Samoilov OYa. Discuss Faraday Soc 1957;24:141.

[28] Valente AJM, Polishchuk AYa, Lobo VMM, Geuskens G. Eur Polym J 2002;38:13.

[29] Baker JP, Hong LH, Blanch HW, Prausnitz JM. Macromolecules 1994;27:1446. 